\title{
Impulse Radio UWB Signal Detection Based on Compressed Sensing
}

\author{
T. Zou, Y.M. Li, T. Li, W.H. Xu, Y.Z. Yan \\ Institute of Communication Technology, \\ Ninbo University \\ Ningbo, China
}

\begin{abstract}
The extremely high sampling rate is a challenge for ultra-wideband (UWB) communication. In this paper, we study the compressed sensing (CS) based impulse radio UWB (IR-UWB) signal detection and propose an IR-UWB signal detection method based on compressive sampling matching pursuit (CoSaMP) algorithm. The proposed approach relies on the fact that UWB received signal is sparse in the time domain. The new method can significantly reduce the sampling rate required by the detection and, moreover, when comparing with the existing matching pursuit (MP) based detection method, it provides a better performance in case of the low signal-to-noise ratio. Simulation results demonstrate the effectiveness of the CoSaMP algorithm used in IR-UWB signal detection.
\end{abstract}

Keywords-IR-UWM; compressed sensing; CoSaMP; MP; signal detection

\section{INTRODUCTION}

UWB is one of the key technologies in the short-range broadband wireless communication. With the characteristics of high data rates, low power, and low cost, UWB can be applied to many scenarios such as high-speed short-range wireless personal area networks (WPAN), ranging, positioning, monitoring, and wireless sensor networks (WSN)[1]. In some applications mentioned above, UWB signal detection is a very important component. Hence there is a need to well study the UWB signal detection to make it more practical.

However, when using the traditional algorithm for UWB signal detection, a very high sampling rate is required according to Shannon-Nyquist sampling theorem for the UWB signal's high bandwidth that is up to several gigahertzes. This is difficult to implement with a practical analog-to-digital converter (ADC) [2]. The emerging theory of CS enables the reconstruction of sparse or compressible signals from a small set of random measurements. If adopted by the signal detection, the CS theory may make the sampling rate much lower than the Nyquist rate. The authors in [3] and [4] proved that it is effective to detect signal by processing the sampling values of compressed sensing directly. Literature [5] proposed a MP based signal detection method. A UWB signal detection method based on MP algorithm was developed in [6]. Whereas in a circumstance with low signal-to-noise ratio, the performance of MP is not good. Thus, it leaves room for improvement. Furthermore, in order to reach a high performance, the number of mixer-integrators employed by the receiver is too large to be realized.

In this paper, we propose a CoSaMP [8] algorithm based IR-UAB signal detection. Without other extra processes, the algorithm is formed from extracting information directly from sampling values acquired by CS. The complexity of the proposed detection method is reduced dramatically when comparing with the GLRT detector. Furthermore, computer simulation results are provided to verify the performance of the proposed method, which show that the performance of the new method is superior to that of the MP, especially in low signal to noise ratio.

\section{COMPRESSED SENSING BACKGROUND}

$\mathrm{CS}$ is a technology that can recover the high-dimensional signals from the low-dimensional and sub-Nyquist sampling data with the prior information that the signals are sparse or compressible [9]. The mathematical model of CS can be described as follows.

$$
\mathbf{y}=\mathbf{A} \mathbf{x}
$$

where $x \in R^{N \times 1}$ is a signal which can be sparsely represented in a basis matrix $\varphi=\left\{\varphi_{1}, \varphi_{2}, \cdots, \varphi_{N}\right\} \in R^{N \times N}$, that is,$x=\varphi \theta$, the vector $\theta=\left[\theta_{1}, \theta_{2}, \cdots, \theta_{N}\right]^{T} \in R^{N \times 1}$ consists of $\mathrm{K}(\mathrm{K}<<\mathrm{N})$ nonzero elements (we often say that $\theta$ is $\mathrm{K}$-sparse). The vector $A \in R^{M \times N} \quad(\mathrm{~K}<\mathrm{M}<<\mathrm{N})$ is a random measurement matrix that is uncorrelated with $\varphi$. $y \in R^{M \times 1}$ denotes the $\mathrm{M}$ samples obtained by CS. Our purpose is to recover the sparse coefficient vector $\theta$ from the $\mathrm{M}$ samples, and then multiply it by the basis matrix ${ }^{\varphi}$, thus recovering the original signal $x$.

In order to figure out the sparse coefficient vector $\theta$, we need to find the solution to the following $l_{0}$ norm optimization problem [10]

$$
\widehat{\theta}=\arg \min \|\theta\|_{0} \quad \text { s.t. } \quad y=A \varphi \theta
$$

Unluckily, solving the optimization problem (2) is prohibitively complex for it's a NP-hard nonconvex optimization problem. A modified problem is to replace the $l_{0}$ restrict with the ${ }^{l}$ restrict

$$
\widehat{\theta}=\arg \min \|\theta\|_{1} \quad \text { s.t. } \quad y=A \varphi \theta
$$


This optimization problem transforms (2) into a convex optimization problem which can be easily solved with linear programming.

\section{IMPULSE RADIO UMB THEORY}

The US Federal Communications Commission (FCC) provided a definition that a signal can be classified as an UWB signal if its fractional bandwidth is greater than 0.2 or its bandwidth is $500 \mathrm{MHz}$ or more [1]. According to this definition, there may be several ways to generate UWB signals, among which impulse radio is the most common method. In this paper, we focus on the impulse radio UWB (IR-UWB) signal.

Owing to different approaches are employed to generate the pulse train, the UWB systems can be divided into two main categories: time hopping UWB(TH-UWB) and direct sequence UWB(DS-UWB). To take a specific case, we will discuss the PAM-DS-UWB signal and its detection in the following. A block diagram of the PAM-DS-UWB transmitter [1] is shown in Fig. 1.

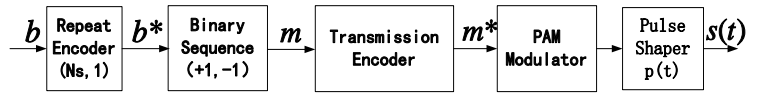

FIGURE I. BLOCK DIAGRAM OF PAM-DS-UWB TRANSMITTER

In Fig. $1, b$ is a binary sequence to be sent and generated at a rate of $R_{b}=1 / T_{b}$ (bits/s). After passing through the repeat encoder, every bit of the sequence $b$ is repeated $N_{s}$ times, therefore we get a new sequence $b^{*}$ where $b^{*}$ has a rate of $R_{c b}=N_{s} / T_{b}=1 / T_{s}$ (bits/s). Then the second system of the block diagram converts $b^{*}$ into a sequence $m$ that contains two kinds of elements, +1 and -1 . The conversion equation of this is $m_{j}=2 b_{j}^{*}-1(-\infty<j<+\infty)$. When the sequence $m$ enters the transmission encoder, $a$ denotes a binary zero correlation duration (ZCD) code composed of \pm 1 's is applied to it [11] and the output of the transmission encoder is a new sequence $m^{*}$, which can be expressed as

$$
m^{*}=\left(\cdots, m_{0} a_{0}, m_{0} a_{1}, \cdots, m_{j} a_{j}, \cdots\right)=\left(\cdots, m_{0}^{*}, m_{1}^{*}, \cdots, m_{j}^{*}, \cdots\right)
$$

The period of the ZCD code $a=\left(\cdots, a_{0}, a_{1}, \cdots, a_{j}, a_{j+1}, \cdots\right)$ is $N_{p}$, we often assume that $N_{p}=N_{s}$ (a more general hypothesis is that $N_{p}$ is an integer multiple of $N_{s}$ ). The rate of sequence $m^{*}$ is $R_{c}=N_{s} / T_{b}=1 / T_{s}$ (bits/s). Next, the sequence $m^{*}$ goes into the PAM modulator, and a sequence of unit pulses (Dirac pulses $\delta(t)$ ) located at times $j T_{s}$ are generated by the PAM modulator [11]. The rate of the sequence of unit pulses is $R_{p}=N_{s} / T_{b}=1 / T_{s}$ (pulses/s). At last, the output of the PAM modulator passes through the pulse shaper, whose impulse response is $p(t)$. The duration of $p(t)$ is $T_{m}$, and $T_{m}<<T_{s}$. Thus, we get the final output signal $s(t)$, which is given by

$$
s(t)=\sum_{j=-\infty}^{+\infty} m_{j}^{*} p\left(t-j T_{s}\right)
$$

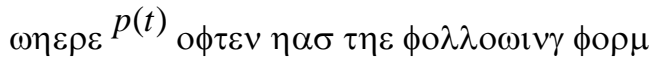

$$
p(t)=\left(1-4 \pi \frac{t^{2}}{\alpha^{2}}\right) e^{-\frac{2 \pi t^{2}}{\alpha^{2}}}
$$

where $\alpha^{2}=4 \pi \sigma^{2}$ is the pulse shaper factor, and $\sigma^{2}$ is the variance.

In practice, A PAM-DS-UWB transmitter's parameters set by the user are: the average transmit power Pow, the number of bits generated by the binary source numbits, the sampling frequency $f_{c}, N_{p}, T_{s}, N_{s}, T_{m}$, and $\alpha^{2}$ [1].

Fig. 2 shows an example of the PAM-DS-UWB signal. From this figure we can see that PAM-DS-UWB signal presents an intuitive sparse characteristic in the time domain. That is, the signal has a few nonzero values. For a fixed and known PAM-DS-UWB transmitter, the number of the nonzero values (the so-called sparsity level, denoted as $S$ ) can be calculated using

$$
s=\text { numbits } * N_{s} * T_{m} * f_{c}
$$

Thus, according to the Section II, the basis matrix of the PAM-DS-UWB signal can be an identity matrix.

Based on the above, we can apply the CS theory into the PAM-DS-UWB signal detection.

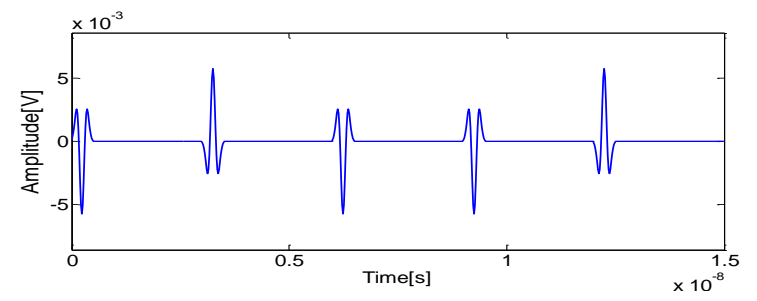

FIGURE II. PAM-DS-UWB SIGNAL

\section{CS BASED IR-UWB SIGNAL DETECTION}

\section{A. The Signal Detection Model}

We implement the detection by distinguishing between the following two hypotheses

$$
H_{0}: y=A n \quad H_{1}: y=A(x+n)
$$

where $x \in R^{N \times 1}$ denotes the PAM-DS-UWB signal to be detected, and $n \sim N\left(0, \sigma^{2} I_{N}\right)$ is the independent and 
identically distributed additive white Gaussian noise. $A \in R^{M \times N}(\mathrm{M}<<\mathrm{N})$ is a known random measurement matrix, and $y \in R^{M \times 1}$ is the sample obtained by the detector. Next, we let

$P_{d}=\operatorname{Pr}\left(H_{1}\right.$ chosen when $H_{1}$ true $)$ and $P_{f}=\operatorname{Pr}\left(H_{1}\right.$ chosen when $H_{0}$ true $)$

denote probability of detection and probability of false alarm respectively [3].

\section{B. CoSaMP based IR-UWB signal detection algorithm}

In this section, we proposed a CoSaMP algorithm [8] based sparse signal detection. The procedure of the algorithm is as follows:

Let $\Phi \in R^{M \times N}$ denote the measurement matrix with restricted isometry constant $\delta_{2 s} \leq c$ ( $c$ is a constant), $u$ denote the noisy sample vector. Furthermore, the sparsity level is $s$, and the output target signal $a$ is an $s$-sparse approximation.

Initialize the approximation $a_{0}=0$ and residual $v=u$. Initialize the iteration counter $k=1$.

Find a proxy $y=\Phi^{*} v$ for the current residual and locate the $2 s$ largest columns of the proxy

$$
\Omega=\operatorname{supp}\left(y_{2 s}\right)
$$

where $\operatorname{supp}\left(y_{2 s}\right)$ means the index set of the $2 s$ largest columns of ${ }^{y}$.

3. Merge the index set of the newly identified components with that of the largest components of the current approximation $T=\Omega \bigcup \operatorname{supp}\left(a_{k-1}\right)$

Solve a least squares problem to make an estimation of the signal $\left.b\right|_{T}=\Phi_{T}^{\dagger} u ;\left.b\right|_{T^{c}}=0$

where $\Phi_{T}^{\dagger}=\left(\Phi_{T}^{*} \Phi_{T}\right)^{-1} \Phi_{T}^{*}$. For an arbitrary $b \in R^{N \times 1}$, assume that $T$ is a subset of $\{1,2, \cdots, N\}$, we define $\left.b\right|_{T}= \begin{cases}b_{i}, & i \in T \\ 0, & \text { otherwise }\end{cases}$

5. Reserve the $s$ largest components in the approximation obtained by the step 4 to produce a new approximation $a_{k}=b_{s}$

6. Update the residual $v=u-\Phi a_{k}$

7. $k=k+1$, if $\|v\|_{2} \leq \varepsilon$, where $\varepsilon$ is a known constant, then go to step 2; or else, go to step 8 .

8. If $\|a\|_{\infty}>\lambda$, where $\lambda$ is a threshold value, detect ${ }^{H_{1}}$; otherwise, choose $H_{0}$.

\section{Simulation}

In this section, the performances of the CoSaMP and the MP detection algorithm are compared. First, we set the parameters of the PAM-DS-UWB transmitter as follows: Pow $=-30(\mathrm{dBm})$, numbits $=2, f_{c}=50 e 9(\mathrm{~Hz}), N_{p}=10(\mathrm{~s})$, $T_{s}=3 e-9(\mathrm{~s}), N_{s}=5, T_{m}=0.5 e-9(\mathrm{~s}), \alpha^{2}=0.25 e-9$. Hence the length of the PAM-DS-UWB signal to be detected is $N=$ numbits $* N_{s} * f_{c} * T_{s}=1500_{\text {[12]. Moreover, according }}$ to (7), the sparsity level of the signal

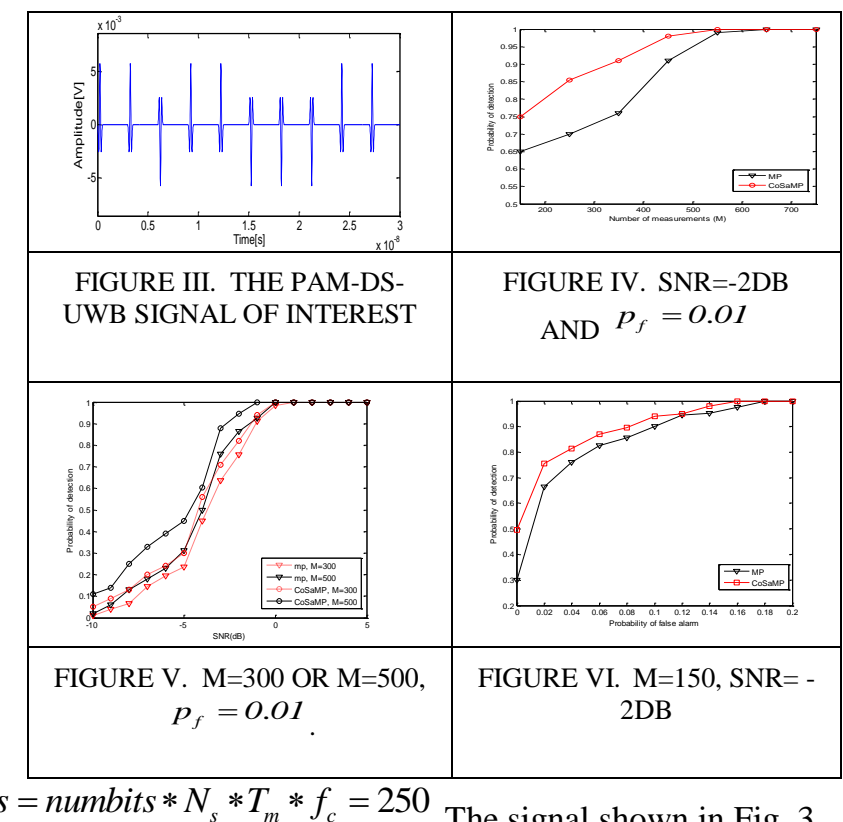

In simulation, we let the measurement matrix $A \in R^{M \times N}$ be an independent and identically distributed Gaussian random matrix with zero-mean and unit variance. Further, the mean and variance of the additive white Gaussian noise $n$ are 0 and 1 , respectively. For the CoSaMP detection algorithm, we set constant $\varepsilon=10^{-5}$. For the MP detection algorithm [5], we set the number of iterations as 10 . Suppose that the probabilities of the two hypotheses are equal, that is $\mathrm{P}_{\mathrm{r}}\left(H_{0}\right)=\mathrm{P}_{\mathrm{r}}\left(H_{1}\right)=0.5$. The probability of detection is the statistic result of 10000 trials.

Fig.4. illustrates $P_{d}$ as a function of $M$, which is the number of measurements. We set the SNR as $-2 \mathrm{~dB}$, and $p_{f}=0.01$. $M$ ranges $[150,750]$. The threshold value $\lambda$ and the threshold value of MP algorithm are both chosen by Monte Carlo simulations [5]. The number of Monte Carlo simulations is 2000. If we use the traditional detection algorithm, the number of measurements should be $N=$ numbits $* N_{s} * f_{c} * T_{s}=1500$ according to the ShannonNyquist sampling theorem. As we can see from this figure, the proposed method can acquire a very high probability of detection at about $20 \%$ of the Nyquist rate. What's more, in 
the same condition, the proposed method is superior to the MP based sparse signal detection method.

Fig. 5 shows $P_{d}$ as a function of the SNR, which ranges [$10,5]$. We consider $M=300$ and $M=500$ respectively. Let $p_{f}=0.01$, According to Fig. 5, we can see that the performance of the CoSaMP detection algorithm is better than that of the MP detection algorithm when the SNR is less than $1 \mathrm{~dB}$.

Fig.6 illustrates $P_{d}$ as a function of $P_{f}$, which ranges [0, $0.2]$. Let $M=150$ and $S N R=-2 d B$. As we can see from Fig.6, the CoSaMP detection algorithm is superior to the MP detection algorithm in this situation.

\section{CONCLUSION}

In this paper, we present a CS based IR-UWB signal detection model and an IR-UWB signal detection algorithm based on CoSaMP. Compared with the GLRT based model, the proposed model is easier to realize. The new detection algorithm solves the detection problem by directly processing the sampling values obtained by CS. It is proved that the proposed algorithm can detect the IR-UWB signal at a rate much lower than the Nyquist rate. Numeric simulations show that the new algorithm yields performance gains over the MP based detection algorithm in case of the low SNR.

\section{REFERENCES}

[1] M. G. Di Benedetto and G. Giancola. Understanding Ultra Wide Band Radio Fundamentals, ser. Prentice-Hall Communications Engineering and Emerging Technology. Englewood Cliffs, NJ: Prentice-Hall, 2004.

[2] G. Shi, J. Lin, X. Chen, et al. UWB Echo Signal Detection With UltraLow Rate Sampling Based on Compressed Sensing. IEEE Transactions on Circuits and Systems, 2008, 4(55):379-383.

[3] M. A. Davenport, M. B. Wakin, R. G. Baraniuk. Detection and Estimation with Compressive Measurements. Houston Tx USA: Rice ECE Departments, 2006:3-13.

[4] J. Haupt, R. Nowak. Compressive Sampling for Signal Detection. IEEE International Conference on Acoustics, Speech, and Signal Processing(ICASSP), Honolulu, Hawaii, April 2007,1509-1512.

[5] M.F. Duarte, M.A. Davenport, M.B. Wakin, et al. Sparse Signal Detection from Incoherent Projections. IEEE International Conference on Acoustics, Speech, and Signal Processing(ICASSP), Toulouse, France, May 2006, 305-308.

[6] J. L. Paredes, G. R. Arce, Z. Wang. Ultra-Wideband Compressed Sensing: Channel Estimation. IEEE Journal of Selected Topics in Signal Processing, 2007,1(3): 383-394.

[7] Z. Wang, G. R. Arce, J. L. Paredes, et al. Compressed Detection for Ultra-Wideband Impulse Radio. IEEE 8th Workshop on Signal Processing Advances in Wireless Communications, Helsinki, Finland, June 2007, 1-5.

[8] D. Needell, J. A. Tropp. CoSaMP: Iterative Signal Recovery from Incomplete and Inaccurate Samples. Applied and Computational Harmonic Analysis, 2009, 3(26): 301-321.

[9] L. Jiao, S. Yang, F. Liu, et al. Development and Prospect of Compressive Sensing. Acta Electronia Sinica, 2011, 7(39): 1651-1662.

[10] P. Zhang, Z. Hu, R.C. Qiu, et al. A Compressed Sensing Based UltraWideband Communication System. IEEE International Conference on Communications, June 2009, 1-5.

[11] E.C. Kim, S. Park, J.S. Cha, et al. Improved Performance of UWB System for Wireless Body Area Networks. IEEE Transactions on Consumer Electronics, 2010, 3(56):1373-1379.
[12] L. Shi. Applied Research on Compressed Sensing for Ultra Wideband Channel Estimation, Ph.D. Thesis, Beijing University of Posts and Telecommunications, 2011. 